\title{
Choroidal Neovascularization and Haller Vessel Morphology Associated with Vision and Treatment Number after 1 Year in Age-related Macular Degeneration
}

\author{
Seungmin Kim, Hyungwoo Lee, Hyewon Chung, Hyung Chan Kim \\ Department of Ophthalmology, Konkuk University Medical Center, Konkuk University School of Medicine, Seoul, Korea
}

\begin{abstract}
Purpose: The present study aimed to quantify various factors of vessel morphology, including vessel diameter, length, and complexity (fractal dimension and lacunarity) of both choroidal neovascularization (CNV) and en face Haller vessels using optical coherence tomography angiography (OCTA) and en face structural optical coherence tomography in typical neovascular age-related macular degeneration (nAMD) and polypoidal choroidal vasculopathy (PCV) and to identify factors associated with visual acuity (VA) loss and number of injections within a year after the day of OCTA.

Methods: We retrospectively analyzed 43 eyes of nAMD patients and 33 eyes of PCV patients whose OCTA was performed at least 12 months after an initial anti-vascular endothelial growth factor treatment. Quantitative parameters, including vessel area, vessel diameter, vessel length, fractal dimension, and lacunarity were analyzed from en face images of CNV and Haller vessels. Clinical information, including logarithm of the minimum angle of resolution visual acuity and injection number of anti-vascular endothelial growth factor were acquired after 12 months from OCTA date. Using logistic regression analyses, parameters associated with logarithm of the minimum angle of resolution VA loss of 0.2 or more (VA loss group) and a number of injections of four or more (unstable group) after 12 months were analyzed.

Results: In typical nAMD, the VA loss group was associated with a smaller number of intersections of Haller vessels. The unstable group was associated with an increased lacunarity of CNV in typical nAMD. In PCV, both VA loss and unstable groups were associated with a higher maximal diameter of Haller vessels.

Conclusions: VA loss and injection number of nAMD and PCV 12 months after OCTA imaging were associated with different morphological parameters of CNV and Haller vessels. Therefore, quantitative analyses of both CNV and Haller vessels from OCTA and en face optical coherence tomography might provide prognostic information about visual outcome and injection frequency within 12 months after OCTA imaging.
\end{abstract}

Key Words: Choroidal neovascularization, Haller vessel, Neovascular age-related macular degeneration, Optical coherence tomography angiography, Polypoidal choroidal vasculopathy

Received: June 1, 2021 Final revision: June 22, 2021

Accepted: August 2, 2021

Corresponding Author: Hyung Chan Kim, MD, PhD. Department of Ophthalmology, Konkuk University Medical Center, 120-1 Neungdongro, Gwangjin-gu, Seoul 05030, Korea. Tel: 82-2-2030-8198, Fax: 82-22030-5273, E-mail: eyekim@kuh.ac.kr
Neovascular age-related macular degeneration (nAMD) is emerging as a major cause of blindness in adults [1]. Polypoidal choroidal vasculopathy (PCV) is considered a variant of nAMD, characterized by a network of branching choroidal vessels with polyp-like aneurysmal dilations, 
and is also known to be common in Asians [2,3]. PCV presents as a form of pachychoroid spectrum disease which is characterized by a thickened choroid, and is also known to exhibit a locally thickened choroidal vessel (pachyvessel) even in the absence of increased choroidal thickness [4].

The recently introduced optical coherence tomography angiography (OCTA) has enabled non-invasive detailed observation of choroidal neovascularization (CNV) morphology. Researchers have identified various quantitative factors for vessel morphology, including vessel area, fractal dimension, and endpoint density, and have linked these factors with visual acuity or the number of injections [5-9]. However, the difference in CNV morphology between typical nAMD and PCV has not been well studied.

The CNV is originated form choroid, therefore, considering the status of both choroid and CNV might be important to understand the pathologic environment. Pachyvessels are believed to be closely associated with the atrophy of choriocapillaris and the development of focal disruptions in the retinal pigment epithelium and Bruch's membrane, which lead to the development of CNV [2]. En face optical coherence tomography (OCT) is a method for observing Haller vessels in frontally, making it possible to obtain a detailed vascular morphology of Haller vessels, including locally changed vascular thickness, branch length, and complexity [9-11]. A previous study which used both OCTA and en face structural OCT showed that CNV morphology and en face images of Haller vessels were associated with visual prognosis and the need for additional injection of anti-vascular epithelial growth factor (VEGF) after 6 months [11]. However, this previous study was limited by its included patients with short-term follow-up as 6 months.

Hence, in the present study we aimed to quantify various factors of vessel morphology, including vessel diameter, length, and complexity (fractal dimension and lacunarity) of both CNV and Haller vessels using OCTA and en face OCT which were performed at least 12 months after an initial treatment in typical nAMD and PCV. Then we identified morphologic factors of both CNV and Haller vessels associated with visual acuity (VA) loss and number of injections within a year after the day of OCTA.

\section{Materials and Methods}

\section{Participants}

This study adhered to the tenets of the Declaration of Helsinki and was approved by the ethics committee of the institutional review board of Konkuk University Medical Center (2020-07-048). This study is a retrospective study based on the medical records of patients who visited the Konkuk University Medical Center between January 1, 2017 and December 31, 2019 and were diagnosed with AMD and monitored for at least 1 year from the time of the initial treatment. Informed consent was waived due to the retrospective nature of the study using medical records. Patients received anti-VEGF treatments, including ranibizumab or aflibercept, following a pro re nata regimen, which was administered at the discretion of the treating physician. Furthermore, the included patients had both OCTA and en face structural OCT images at least 1 year after the time of the initial treatment and should have received further follow-up for at least 1 year from the day of OCTA imaging (OCTA date), and had information on VA after 1 year and the number of injections during the 1-year follow-up.

In all subjects, fluorescent angiography and indocyanine green angiography (ICGA) were performed at the initial visit and visual acuity, intraocular pressure, best-corrected visual acuity, fundus examination, and fundus photography were performed at each visit. OCTA and en face structural OCT was performed after 1 year from the initial visit. Typical nAMD was defined as nAMD including any type of CNV (type 1 or 2) confirmed by fluorescent angiography and ICGA. The diagnosis of PCV was based on the detection of elevated orange-red lesions on fundal examination and the presence of polypoidal lesions on ICGA. We excluded patients with high myopia (<-6 diopters), images obscured by media opacity, significant cataract progression without surgery during follow-up, other combined retinal diseases, or geographic atrophy. We also excluded patients with poor OCTA images that had projection artifacts and artifacts from eye movement that resulted in the breakage of vessel morphology. Additionally, poor images of en face Haller vessels were excluded when vague demarcation of vessels from the stroma was observed. 

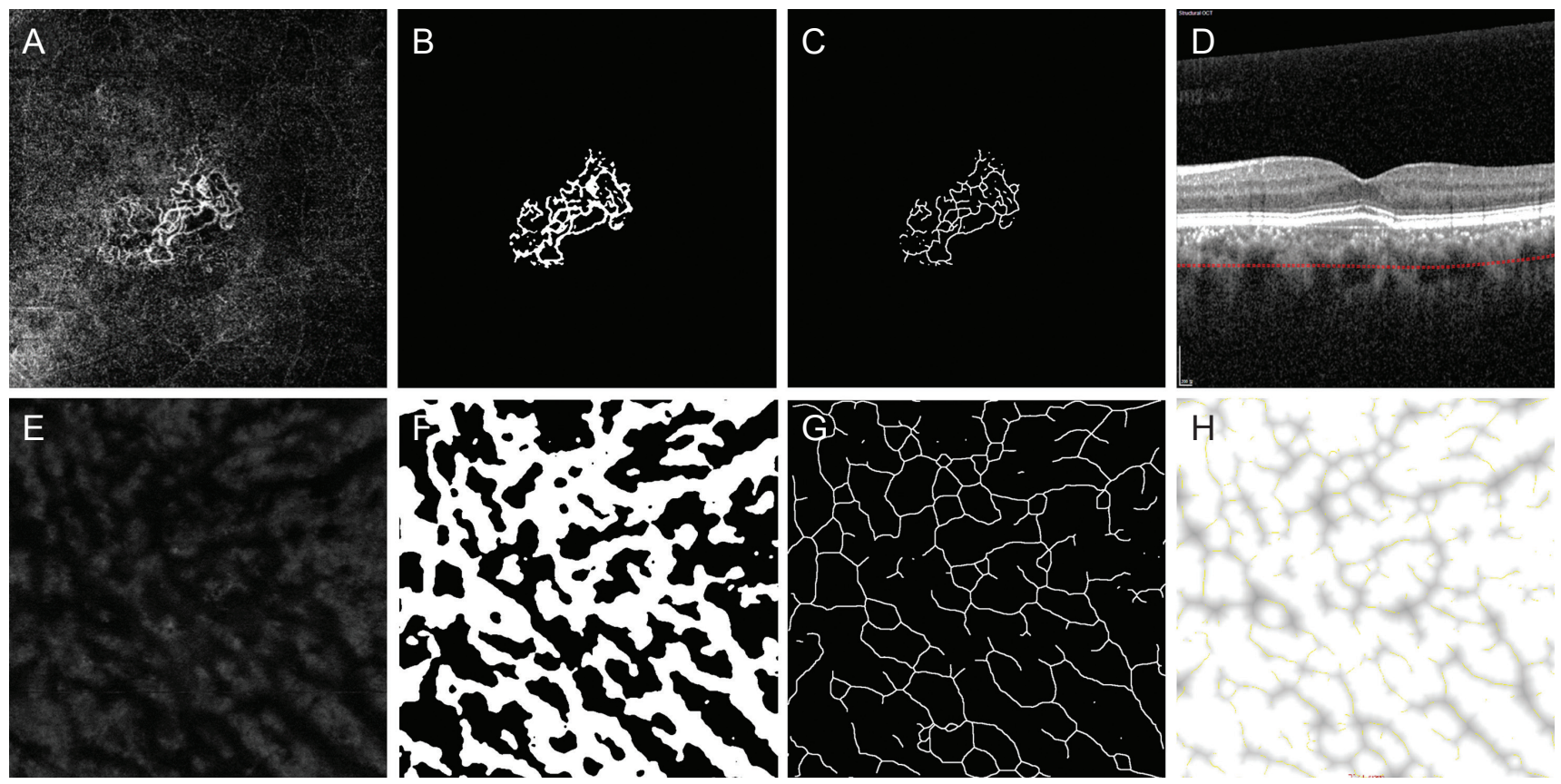

Fig. 1. Quantitative measurement of choroidal neovascularization and Haller vessels. (A) Optical coherence tomography angiography image showing choroidal neovascularization. (B) From binarized image using Otsu filter, vessel area was measured. (C) Binarized image was processed to skeletonized image using ImageJ, and following parameters were calculated using DiameterJ plugin; total length, branch length, and the number of intersections. (D) En face image of Haller vessel was obtained by adjusting the thickness of the choriocapillaris slab to $10 \mu \mathrm{m}$ and locating the slab (red dashed line) to span the center of Haller vessel in B-scans. (E) Acquired en face image of Haller vessel. (F) In the same manner used in choroidal neovascularization, Otsu filter was applied to make binarized image and Haller vessel area was measured. (G) Binarized Haller vessel image was skeletonized, and the total length, branch length, and the number of intersections were calculated using DiameterJ plugin. (H) Finally, the diameter-associated parameters of vessel (mean, median, and maximum) were calculated from the binarized image of $(\mathrm{F})$.

\section{Acquisition of CNV and Haller vessel images from OCTA and en face OCT}

OCTA images and en face structural OCT images were acquired using Spectralis OCT (Spectralis OCT2+HRA; Heidelberg Engineering, Heidelberg, Germany), and the scanned area was set as $6 \times 6 \mathrm{~mm}$. To obtain en face images of $\mathrm{CNV}$, the choriocapillaris slab was adjusted to cover the upper and lower borders of the CNV to depict the whole CNV morphology, as much as possible, with minimal noise signals. When the branches of the CNV were discontinuous or abruptly cut off, the lower border of the preset $\mathrm{CC}$ slab was moved to include the intact $\mathrm{CNV}$. When the proper boundary of the slab was ambiguous, Bscan images were obtained to ensure the integration of the entire CNV complex between the two slabs. The gathered images were then additionally reviewed to determine whether they were of sufficient image quality for further image analyses.
En face images of the Haller's layer were obtained by adjusting the thickness of the choriocapillaris slab to 10 $\mu \mathrm{m}$ and locating the slab to span the center of the Haller vessel in B-scans (Fig. 1A-1H). When the contour of the slab could not span the central line of the entire Haller vessel, the priority was set as the macular area.

\section{Image processing of acquired images of CNV and Haller vessel}

The outer boundary of CNV was manually outlined by the researcher (SK) and confirmed by retinal physicians (HL and HCK) using ImageJ (National Institute of Health, Bethesda, MD, USA) and the area inside the boundary was measured to calculate 'CNV area' [12]. After binarization of the CNV vessels by the Otsu method, the vessel area was calculated using ImageJ. After skeletonization of the binarized vessels, the mean and standard deviation of the total length and the branch vessel length were obtained. To 


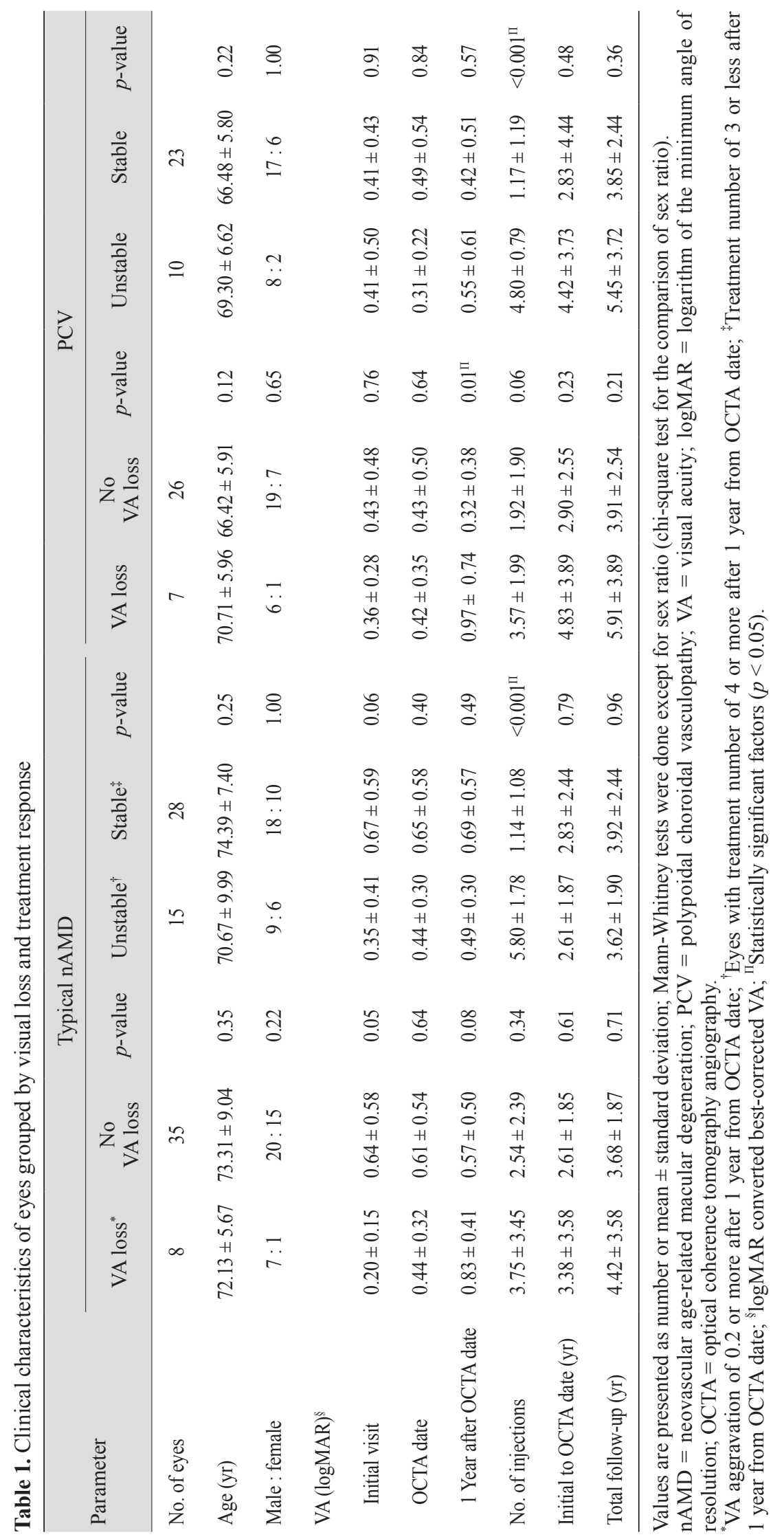


quantify diameter-related characteristics of vessels, the DiameterJ plugin of ImageJ was applied to the binarized image of $\mathrm{CNV}$, and the mean, standard deviation, and maximum diameters of a CNV were calculated (Fig. 1) [13]. To quantify vessel complexity such as fractal dimension and lacunarity, we used the FracLac plugin of ImageJ [14]. While fractal analysis assesses the complexity of a fractal object, lacunarity provides an indication of the empty space and degree of vessel inhomogeneity [15].

\section{Grouping patients according to visual loss and number of injections}

Clinical outcomes were assessed according to two major criteria: (1) visual loss and (2) number of injections after 12 months from the day of OCTA and en face OCT. Regarding visual loss, patients were divided into two groups: a visual loss group, including patients whose logarithm of the minimum angle of resolution ( $\log$ MAR) was aggravated by more than 0.2 from baseline, and other patients ("no VA loss group"). Regarding the number of injections, patients were divided into two groups: the "unstable group" who had received four or more injections for 1 year after the OCTA date, while the other patients were defined as the "stable group."

\section{Statistical analysis}

PASW Statistics ver. 18.0.0 (SPSS Inc., Chicago, IL, USA) was used for all statistical analyses. The MannWhitney test was performed to compare mean values between the two groups, and the chi-square test was used to compare the ratios of men to women. The associations of morphologic parameters with visual loss and number of injections were analyzed using univariate logistic regression analysis. Age, sex, and VA on OCTA data were adjusted in the regression analysis for the VA loss versus no VA loss group. Age and sex were adjusted in the regression analysis for the unstable versus stable group. Results with $p$-values less than 0.05 were considered statistically significant. Since only one statistically significant parameter was found among multiple parameters in univariate regression analysis, multivariate regression analysis was not performed.

\section{Results}

A total of 76 eyes of 71 patients diagnosed with the wet form of AMD were included in this study (43 eyes of 39 patients were diagnosed with typical nAMD and 33 eyes of 32 patients were diagnosed with PCV). Patients with typical nAMD had a mean age of $73.09 \pm 8.47$ years, while those with PCV had a mean age of $67.33 \pm 6.10$ years. The mean initial $\log$ MAR VA and the mean logMAR VA on the OCTA day were $0.56 \pm 0.55$ and $0.58 \pm 0.50$, respectively, in typical nAMD patients, and $0.41 \pm 0.45$ and 0.43 \pm 0.47 , respectively, in PCV patients. The mean logMAR VA 1 year after the OCTA day was $0.62 \pm 0.49$ in typical nAMD patients and $0.46 \pm 0.54$ in PCV patients, both groups did not show statistical difference in the change in VA. Clinical characteristics according to the subgroups based on VA change (VA loss vs. no VA loss) and injection number (unstable vs. stable) are also shown in Table 1. Age, sex, VA on the OCT date, and total follow-up period were not different between the subgroups (Table 1).

Morphological parameters from CNV and Haller vessels according to VA loss and injection number during 1 year of follow-up are depicted in Table 2. Regarding VA loss after 1 year, the mean branch length of Haller vessel was statistically longer in the typical nAMD group than in the no VA loss group, while CNV showed no difference (Table 2 and Fig. 2A-2H). Regarding the number of injections within 1 year in typical nAMD patients, the unstable group showed higher lacunarity of $\mathrm{CNV}$, while there was no difference in Haller vessel morphology between stable and unstable groups (Table 2 and Fig. 3A-3H). Concerning $\mathrm{PCV}$, there was no significant difference in either CNV or Haller vessels between VA loss and no VA loss groups (Table 2 and Fig. 4A-4H). Regarding the number of injections within 1 year in PCV patients, the unstable group showed a shorter total length of the Haller vessel, while CNV showed no difference between stable and unstable groups (Table 2 and Fig. 4).

Next, we identified the morphologic factors of CNV and Haller vessels associated with VA loss and injection number from the univariate logistic regression analysis (Table 3). Regarding VA loss in typical nAMD, the VA loss group was associated with a lower intersection number of Haller vessels (Table 3). Concerning PCV, the VA loss group was associated with a thicker maximum diameter of the Haller vessel (Table 3). Regarding the injection number in typical 


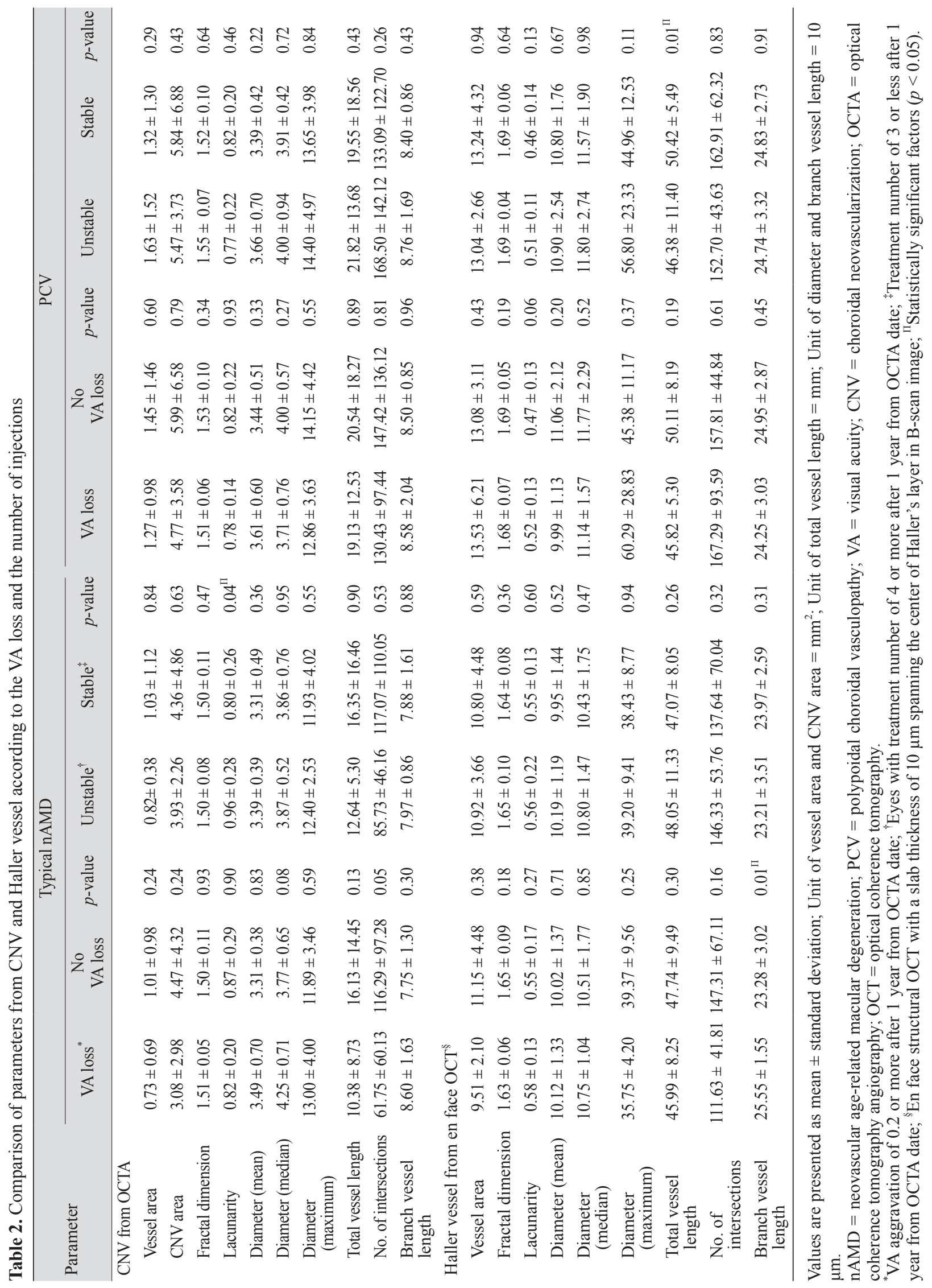



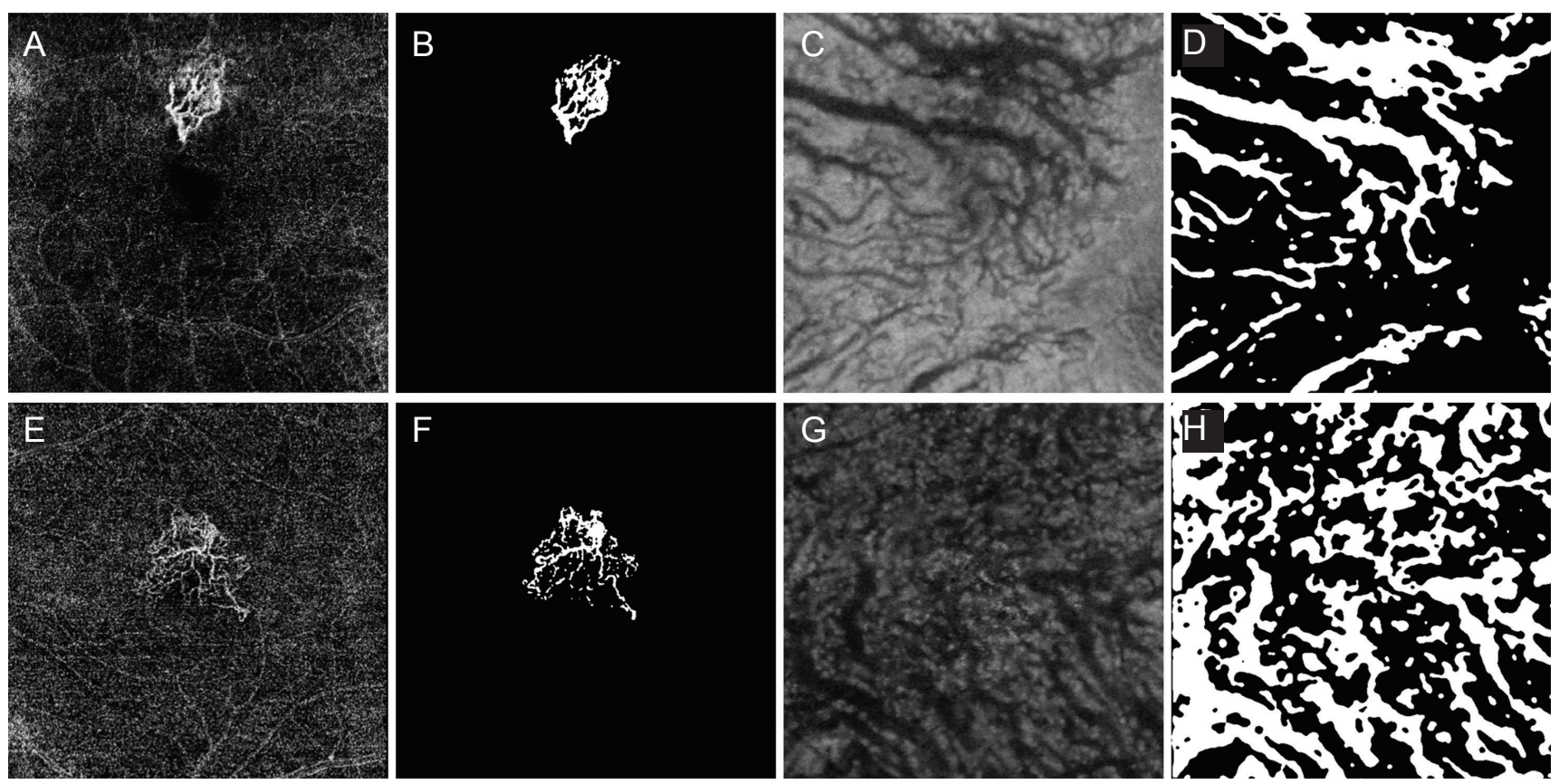

Fig. 2. Choroidal neovascularization (CNV) and Haller vessel morphology in eyes with visual acuity loss (A-D) and no visual acuity loss (E-H) in the typical neovascular age-related macular degeneration group. (A) Optical coherence tomography angiography (OCTA) image obtained in the right eye of a 75-year-old male with visual acuity loss. The follow-up period from the initial visit was 0.76 years. The best-corrected visual acuity (BCVA) on the day of imaging (OCTA date) was $20 / 30$ and the BCVA 1 year after the OCTA date was decreased to $20 / 63$. The total injection number was 2, 1 year after the OCTA date. The CNV area was $1.22 \mathrm{~mm}^{2}$ and the vessel area was $0.44 \mathrm{~mm}^{2}$. (B) Binarized image of the eye is shown in (A). CNV fractal dimension was 1.57 and lacunarity was 0.64 . (C) En face image of Haller vessel obtained in the same patients. The vessel area was $7.11 \mathrm{~mm}^{2}$. The mean diameter was $94.62 \mu \mathrm{m}$ and the maximal diameter was $340.00 \mu \mathrm{m}$. The total length of Haller vessel was $35.46 \mathrm{~mm}$ and the branch vessel length was $271.36 \mu \mathrm{m}$. (D) Binarized image of the eye shown in (C). The number of intersections was low as 65. (E) OCTA image obtained in the left eye of a 61-year-old male without visual acuity loss. The follow-up period from the initial visit was 1.38 years. The BCVA on the OCTA date was $20 / 25$ and the BCVA 1 year after the OCTA date was $20 / 25$. The total injection number was 0,1 year after the OCTA date. The $\mathrm{CNV}$ area was $2.13 \mathrm{~mm}^{2}$ and the vessel area was $0.53 \mathrm{~mm}^{2}$. (F) Binarized image of the eye shown in (E). CNV fractal dimension was 1.53 and lacunarity was 0.79 . Number of intersections was 49. (G) En face image of Haller vessel obtained in the same patients. The vessel area was $18.14 \mathrm{~mm}^{2}$. The mean diameter was $112.72 \mu \mathrm{m}$ and the maximal diameter was $380.00 \mu \mathrm{m}$. The total length of Haller vessel was $62.21 \mathrm{~mm}$ and the branch vessel length was $232.52 \mu \mathrm{m}$. (H) Binarized image of the eye shown in $(\mathrm{G})$. The number of intersections was high as 282 .

nAMD, the unstable group was associated with a higher lacunarity of CNV (Table 3). Concerning PCV, the unstable group was also associated with a thicker maximum diameter of the Haller vessel (Table 3).

\section{Discussion}

In this study, we quantified the morphological characteristics of CNV and Haller vessels in the pretreated patients with typical nAMD and PCV whose follow-up periods were at least 1 year, and analyzed their association with VA loss and injection number after a year. In a previous study which employed both OCTA and en face OCT, the follow-up duration was short (6 months); thus, in the previous study, we attempted to determine vascular parameters associated with longer prognosis, 1 year after OCTA and en face OCT.

In this study, the VA loss group in typical nAMD showed a longer mean branch length of Haller vessels than the no VA loss group (Table 2). According to Poiseuille's rule, longer blood vessels have higher resistance, resulting in decreased blood flow [16]. Therefore, the longer branch length of Haller vessels might have hampered sufficient blood supply and aggravated the ischemia, eventually contributing to visual loss. When the association was analyzed by logistic regression analysis, the VA loss group in typical nAMD was associated with a lower intersection number of 

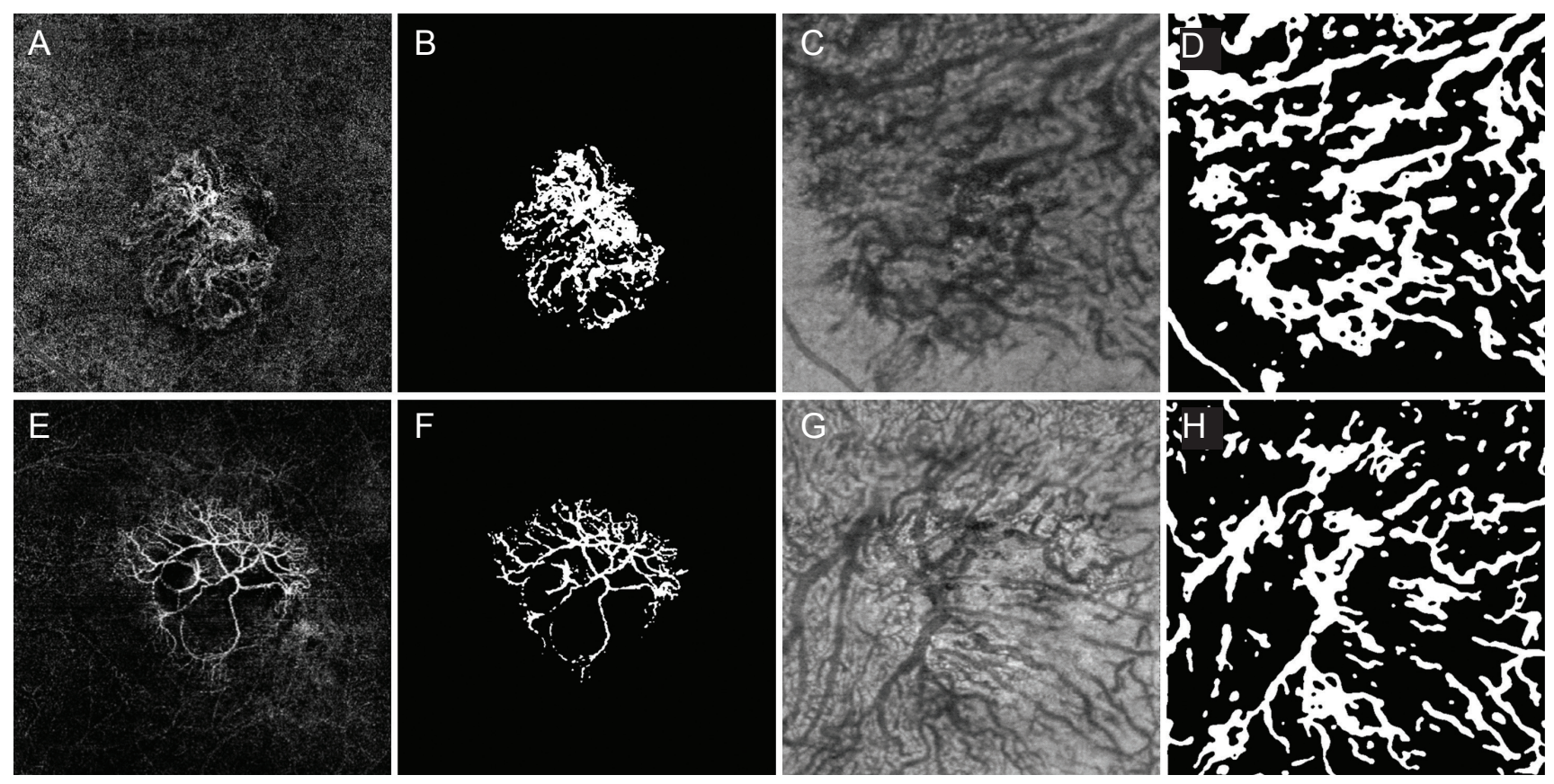

Fig. 3. Choroidal neovascularization (CNV) and Haller vessel morphology in eyes in the unstable group which required four or more injections within 1 year (A-D) and eyes in the stable group (E-H) in the typical neovascular age-related macular degeneration group. (A) Optical coherence tomography angiography (OCTA) image obtained in the left eye of a 72-year-old male with frequent injections. The follow-up period from the initial visit was 2.11 years. The best-corrected visual acuity (BCVA) on the day of imaging (OCTA date) was 20 / 63 and the BCVA, 1 year after the OCTA date was $20 / 63$. The total injection number was 7, 1 year after the OCTA date. The $\mathrm{CNV}$ area was $5.84 \mathrm{~mm}^{2}$ and the vessel area was $1.11 \mathrm{~mm}^{2}$. (B) Binarized image of the eye shown in (A). CNV fractal dimension was 1.53 and lacunarity was high as 1.16. Number of intersections was 122. (C) En face image of Haller vessel obtained in the same patients. The vessel area was $12.31 \mathrm{~mm}^{2}$. The mean diameter was $106.16 \mu \mathrm{m}$ and the maximal diameter was $380.00 \mu \mathrm{m}$. The total length of Haller vessel was $57.26 \mathrm{~mm}$ and the branch vessel length was $235.31 \mu \mathrm{m}$. (D) Binarized image of the eye shown in (C). The number of intersections was 186. (E) OCTA image obtained in the left eye of an 82-year-old male without frequent injections. The follow-up period from the initial visit was 3.72 years. The BCVA on the OCTA date was $20 / 400$ and the BCVA, 1 year after the OCTA date was $20 / 200$. The total injection number was 1,1 year after the OCTA date. The CNV area was $5.84 \mathrm{~mm}^{2}$ and the vessel area was $1.21 \mathrm{~mm}$. (F) Binarized image of the eye shown in (E). CNV fractal dimension was 1.54 and lacunarity was 0.77 which was smaller than that of unstable group. Number of intersections was 125. (G) En face image of Haller vessel obtained in the same patients. The vessel area was $8.97 \mathrm{~mm}^{2}$. The mean diameter was $84.52 \mu \mathrm{m}$ and the maximal diameter was $260.00 \mu \mathrm{m}$. The total length of Haller vessel was $56.00 \mathrm{~mm}$ and the branch vessel length was $226.34 \mu \mathrm{m}$. (H) Binarized image of the eye shown in $(\mathrm{G})$. The number of intersections was 144.

Haller vessels (Table 3). Different parameters were found in the results of mean comparison (longer mean branch length of Haller vessels) and regression analysis (lower intersection number of Haller vessels), and this result might have originated from the different statistical methods between the studies, and the additional adjustment of age, sex, and VA on the OCTA data in the regression analysis. Regarding the prognostic value, the results of regression analysis adjusted for confounding factors are considered more reliable. Concerning the connection between a lower intersection number of the Haller vessel and VA loss, for a Haller vessel at a lower intersection, there might be a lower chance of communication with other vessels. When ischemic threat to the choriocapillaris occurs, the higher inter- section of the Haller vessel might have various routes to supply blood to those areas, even if some interconnecting Haller vessels have a lower ability to convey blood. Therefore, the lower intersection number of Haller vessels might be associated with VA loss after 1 year, as indicated in our study. In a previous study of the patients with the 6 months follow-up, VA loss in typical nAMD was associated with higher lacunarity of Haller vessels [11]. This factor was not significant in the present study. Although the study population in the present study and that in the previous study are different, the common finding is that the Haller vessel was an associated factor, not CNV. Additionally, higher lacunarity implies an inhomogeneous distribution of the gap between Haller vessels. Although a lower intersection 

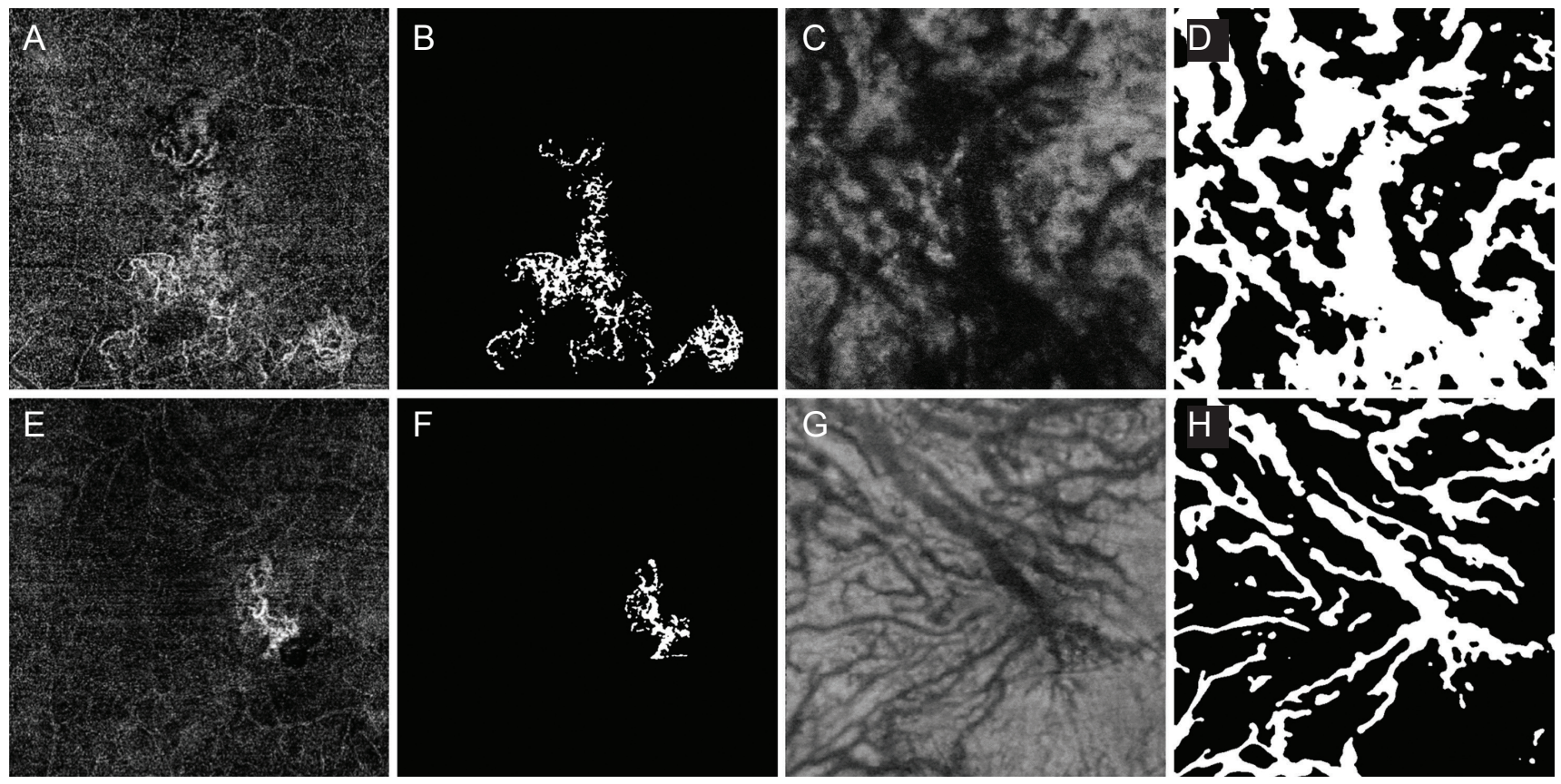

Fig. 4. Choroidal neovascularization (CNV) and Haller vessel morphology in eyes with visual acuity loss as well as unstable which required four or more injection within 1 year (A-D), and no visual acuity loss with stable (E-H) in the polypoidal choroidal vasculopathy group. (A) Optical coherence tomography angiography (OCTA) image obtained in the left eye of a 65-year-old male with visual loss and frequent injections. The follow-up period from the initial visit was 0.92 years. The best-corrected visual acuity (BCVA) on the day of imaging (OCTA date) was $20 / 100$ and the BCVA on the 1 year after the OCTA date was decreased to $20 / 200$. The total injection number was four, 1 year after the OCTA date. The CNV area was $5.43 \mathrm{~mm}^{2}$ and the vessel area was $1.15 \mathrm{~mm}^{2}$. (B) Binarized image of the eye shown in (A). CNV fractal dimension was 1.58 and lacunarity was 0.68 . Number of intersections was 174 . (C) En face image of Haller vessel obtained in the same patients. The vessel area was $14.63 \mathrm{~mm}^{2}$. The mean diameter was $97.97 \mu \mathrm{m}$ and the maximal diameter was high as $680.00 \mu \mathrm{m}$. The total length was $37.75 \mathrm{~mm}$ and the branch vessel length was $227.69 \mu \mathrm{m}$. (D) Binarized image of the eye shown in (C). The number of intersections was 164. (E) OCTA image obtained in the right eye of a 69 -year-old male without significant visual loss and infrequent injections. The follow-up period from the initial visit was 2.47 years. The BCVA on the OCTA date was $20 / 20$ and the BCVA, 1 year after the OCTA date was $20 / 25$. The total injection number was 2,1 year after the OCTA date. The CNV area was 1.13 $\mathrm{mm}^{2}$ and the vessel area was $0.32 \mathrm{~mm}^{2}$. (F) Binarized image of the eye shown in (E). CNV fractal dimension was 1.52 and lacunarity was 0.66. Number of intersections was 26. (G) En face image of Haller vessel obtained in the same patients. The vessel area was $10.83 \mathrm{~mm}^{2}$. The mean diameter was $110.32 \mu \mathrm{m}$ and the maximal diameter was low as $420.00 \mu \mathrm{m}$. The total length of Haller vessel was $51.75 \mathrm{~mm}$ and the branch vessel length was $320.71 \mu \mathrm{m}$. (H) Binarized image of the eye shown in (G). The number of intersections was 141.

number and inhomogeneous gaps do not indicate exactly the same status, higher interconnection might contribute to morphology, such as close stitches, decreasing the chance of an inhomogeneous gap between Haller vessels. Further studies with larger populations might reveal a more representative network structure of Haller vessels associated with VA loss.

Regarding PCV, the VA loss group showed no morphological difference compared to the no VA loss group, but the VA loss group was associated with a thicker maximum diameter of Haller vessel in the regression analysis. Pachyvessels are dilated outer choroidal vasculature structures observed in pachychoroid spectrum diseases, including PCV. Dilated choroidal vessels are considered to be as- sociated with pathological changes in the overlying thin choriocapillaris [17]. Therefore, the thicker maximum diameter of the Haller vessel might indicate poorer function of the choriocapillaris, eventually decreasing vision. Because change in the choriocapillaris was not investigated in this study, further studies combining the Haller vessel and choriocapillaris status are needed. In a previous study of the 6 months follow-up, no factor related to CNV and Haller vessels was associated with VA loss [17]. Although a direct comparison is inappropriate, the morphologic analysis in PCV might be associated with longer term prognosis. Because VA loss measures the deterioration of visual acuity during specific period, previous study restricted to 6 months follow-up could not reveal the possible 


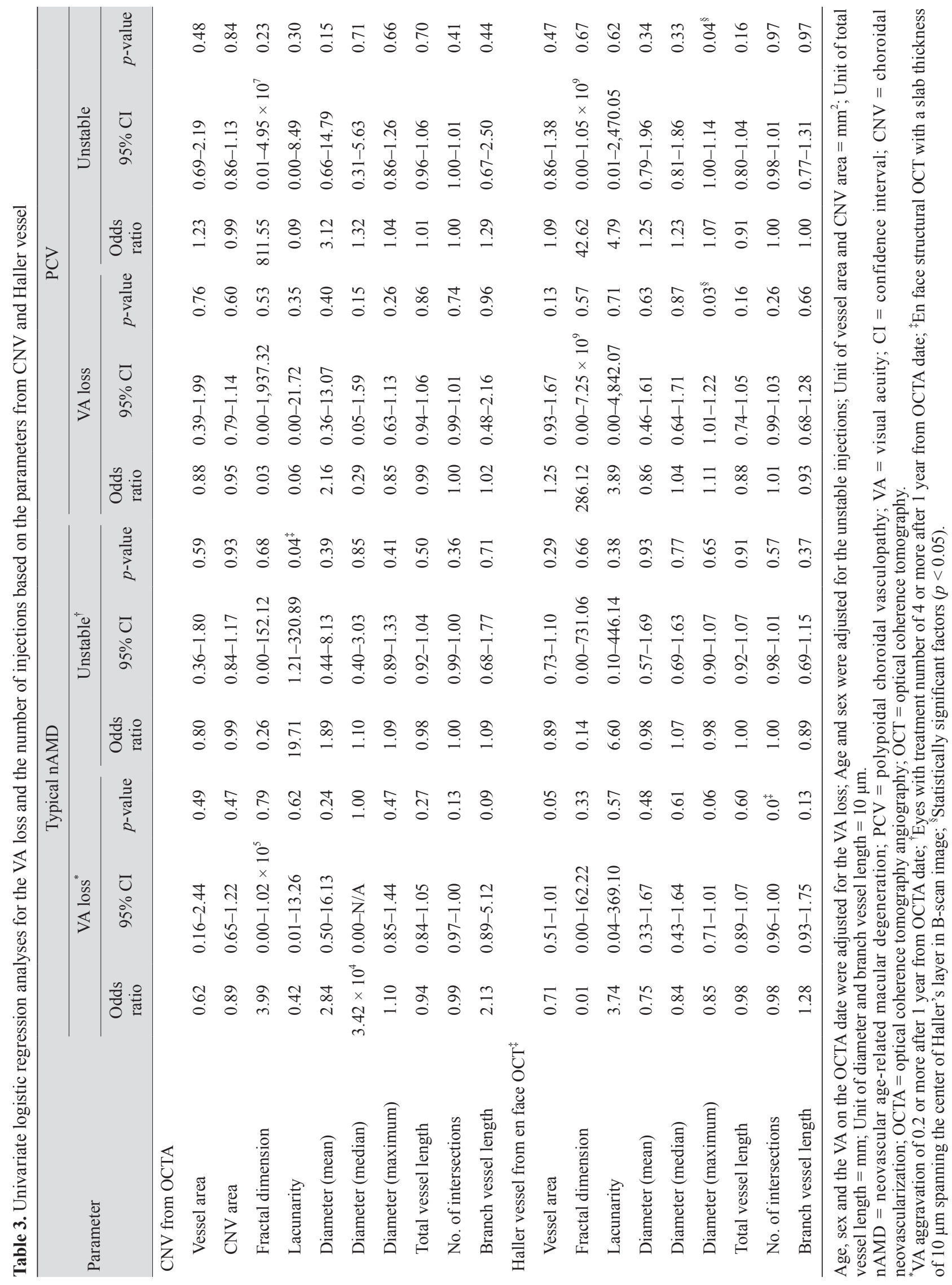


effect from the dilated Haller vessel. VA loss is directly affected by the activity of CNV - the formation of fluid and/ or hemorrhage. This activity of CNV is thought to be also affected by the pathologic change of choriocapillaris, and the dilated Haller vessel is thought to be associated of the thinning and atrophic change of choriocapillaris [17]. Therefore, the VA loss from the dilated Haller vessel seems to be 'indirectly' associated via dysfunctional choriocapillaris and more active $\mathrm{CNV}$, and longer period is needed.

Concerning the injection number, the unstable group in typical nAMD showed higher lacunarity of CNV than the stable group, and unstable group was also associated with higher lacunarity of $\mathrm{CNV}$ in the regression analysis. As discussed earlier, higher lacunarity implies an inhomogeneous distribution of gaps between vessels, implying increased complexity of $\mathrm{CNV}$. As the activity of $\mathrm{CNV}$ might contain more tiny capillaries and budding vessels, lacunarity is expected to increase. Choi et al. [9] showed increased lacunarity in a subgroup of patients among nAMD patients that needed more injections for 1 additional year, but this parameter was not found to be significant in the univariate regression analysis. Although in the study by Choi et al. [9], patients were not divided into typical nAMD and PCV groups, this common finding of higher lacunarity in the unstable group might suggest the prognostic value of $\mathrm{CNV}$ lacunarity on injection number. In a previous study of the 6 months follow-up study, CNV-associated factors were not found, but a thinner Haller vessel diameter was associated with at least one injection [11]. Because the previous study defined poor response as at least one injection within 6 months, it might be more sensitive than our study in which the definition of the unstable group was patients who received four or more injections. To elucidate injection number-associated parameters, a study with a larger population and long-term follow-up might be needed.

In PCV, the unstable group had a shorter total length of the Haller vessel. Logistic regression analysis adjusted for age and sex revealed that the unstable group was associated with a thicker maximum diameter of the Haller vessel. Notably, a thicker maximum diameter of the Haller vessel was significantly associated with VA loss in PCV. As discussed above, increased Haller vessel diameter is suggested to be closely associated with a devastating status of the overlying choriocapillaris and the development of CNV [4].
Therefore, the concomitant significance of thick Haller vessels in both the unstable and VA loss groups supports the pachychoroid hypothesis as the origin of CNV in PCV. On the other hand, in the previous study with short-term follow up with 6 months, we could not find associated morphologic factor in PCV [11]. As discussed in the typical nAMD section above, possible hypothesis is that the difference in the definition of treatment response-poor response as at least one injection within 6 months in the previous study, and four or more injections for 1 year in this study. Furthermore, as discussed in the injection number, injection number and dilated Haller vessel are indirectly connected via dysfunctional choriocapillaris and active $\mathrm{CNV}$, and therefore longer period might be needed.

Among the investigated morphologic factors, fractal dimension of both $\mathrm{CNV}$ and Haller vessel were not found to be associated with any clinical outcomes in both typical nAMD and PCV. Anti-VEGF treatment is known to prune small-caliber vessels; hence, reduction in the complexity of $\mathrm{CNV}$ is anticipated. In previously treated patients, significant reduction in the fractal dimension was observed in quiescent CNV [18]. However, as in our result, fractal dimension might not be sufficient to anticipate the clinical outcomes, while it could explain the current status-quiescent or not. Further study with prospective, larger population might reveal the prognostic value of fractal dimension. Additionally, diameter of $\mathrm{CNV}$ were not found to be associated with any clinical outcomes in both typical nAMD and PCV. We expected that the greater the vessel thickness, the more the vessels are matured and this status might lead to greater resistance to anti-VEGF treatment accompanying more injections. The diameter of $\mathrm{CNV}$ is much thinner than that of Haller vessel, and the current image binarization method and analysis tool (DiameterJ) might not be sufficient to catch the subtle change of CNV diameter. Further study with advanced image processing methodology might unveil the association of CNV diameter with the clinical outcomes.

Recently, Choi et al. [9] found that the density of CNV, an endpoint that was not measured in our study, was associated with injection frequency in previously treated patients with nAMD. Further investigations of other morphologic parameters representing the characteristics of $\mathrm{CNV}$, such as density, may unveil additional predictors of the optimal number of injections required for patients with treatment-naïve nAMD. 
This study has several limitations. First, the device we used was SD-OCT; therefore, limitations exist in the depth of imaging when the choroid is extremely thick. Although we excluded Haller images with poor quality in this study, swept-source OCT might be used to obtain more accurate images of Haller vessels, even when the choroid is extremely thick. Second, whole CNV and Haller vessels could not be fully reflected in the acquired images because adjusting predefined slabs was not perfectly fit to contain the whole CNV or span the central line of the Haller vessel. Further development of the algorithm may improve this limitation. In addition, this study did not include patients with retinal angiomatous proliferation; therefore, the interpretation of the results should be limited to patients with typical nAMD and those with PCV. Furthermore, this study was retrospectively designed and included a small number of patients. Further studies with a prospective cohort might elucidate the clinical implications of information on both $\mathrm{CNV}$ and en face Haller vessels in nAMD, including the nonsignificant factors in this study. Finally, our study design was based on the population who already had been treated by anti-VEGF. Because the majority of patients in the real-world clinic are taking OCTA and en face OCT in the cross-sectional setting, we thought that finding prognostic factor in this setting might be useful. However, still the study on the treatment-naïve patients should be performed in further study because finding the important morphologic factor in the early treatment phase might be crucial for the tailored treatment specific for each patient.

In conclusion, we found that visual loss and treatment number after 1 year were associated with the morphological parameters of CNV and en face Haller vessels in typical nAMD and PCV.

\section{Conflict of Interest}

No potential conflict of interest relevant to this article was reported.

\section{References}

1. Pauleikhoff D. Neovascular age-related macular degeneration: natural history and treatment outcomes. Retina
2005;25:1065-84.

2. Pang CE, Freund KB. Pachychoroid neovasculopathy. Retina 2015;35:1-9.

3. Cheung CM, Lai TY, Ruamviboonsuk P, et al. Polypoidal choroidal vasculopathy: definition, pathogenesis, diagnosis, and management. Ophthalmology 2018;125:708-24.

4. Cheung CM, Lee WK, Koizumi H, et al. Pachychoroid disease. Eye (Lond) 2019;33:14-33.

5. Kuehlewein L, Bansal M, Lenis TL, et al. Optical coherence tomography angiography of type 1 neovascularization in age-related macular degeneration. Am J Ophthalmol 2015;160:739-48.

6. Roberts PK, Nesper PL, Gill MK, Fawzi AA. Semiautomated quantitative approach to characterize treatment response in neovascular age-related macular degeneration: a real-world study. Retina 2017;37:1492-8.

7. Xu D, Davila JP, Rahimi M, et al. Long-term progression of type 1 neovascularization in age-related macular degeneration using optical coherence tomography angiography. Am J Ophthalmol 2018;187:10-20.

8. Coscas F, Cabral D, Pereira T, et al. Quantitative optical coherence tomography angiography biomarkers for neovascular age-related macular degeneration in remission. PLoS One 2018;13:e205513.

9. Choi M, Kim SW, Yun C, Oh J. OCT angiography features of neovascularization as predictive factors for frequent recurrence in age-related macular degeneration. Am J Ophthalmol 2020;213:109-19.

10. Baek J, Kook L, Lee WK. Choriocapillaris flow impairments in association with pachyvessel in early stages of pachychoroid. Sci Rep 2019;9:5565.

11. Lee H, Lee M, Kim MA, et al. Association of treatment response with quantitative changes in choroidal neovascularization and choroidal vessel in neovascular age-related macular degeneration. Retina 2020;40:1704-18.

12. Schneider CA, Rasband WS, Eliceiri KW. NIH image to ImageJ: 25 years of image analysis. Nat Methods 2012;9:671-5.

13. Hotaling NA, Bharti K, Kriel H, Simon CG Jr. DiameterJ: a validated open source nanofiber diameter measurement tool. Biomaterials 2015;61:327-38.

14. Karperien A, Ahammer H, Jelinek HF. Quantitating the subtleties of microglial morphology with fractal analysis. Front Cell Neurosci 2013;7:3.

15. Plotnick RE, Gardner RH, Hargrove WW, et al. Lacunarity analysis: a general technique for the analysis of spatial patterns. Phys Rev E Stat Phys Plasmas Fluids Relat In- 
terdiscip Topics 1996;53:5461-8.

16. Sutera SP, Skalak R. The history of Poiseuille's law. Annu Rev Fluid Mech 1993;25:1-19.

17. Dansingani KK, Balaratnasingam C, Naysan J, Freund KB. En face imaging of pachychoroid spectrum disorders with swept-source optical coherence tomography. Retina
2016;36:499-516.

18. Al-Sheikh M, Iafe NA, Phasukkijwatana N, et al. Biomarkers of neovascular activity in age-related macular degeneration using optical coherence tomography angiography. Retina 2018;38:220-30. 